\title{
Elucidating the underlying causes of oral cancer through spatial clustering in high-risk areas of Taiwan with a distinct gender ratio of incidence
}

Chi-Ting Chiang ${ }^{1}$, Yaw-Huei Hwang ${ }^{2}$, Che-Chun Su³, Kuo-Yang Tsai ${ }^{4}$, Ie-Bin Lian ${ }^{5}$, TzuHsuen Yuan ${ }^{2}$, Tsun-Kuo Chang ${ }^{1}$

${ }^{1}$ Department of Bioenvironmental Systems Engineering, National Taiwan University, Taipei City 106, Taiwan; ${ }^{2}$ Graduate Institute of Occupational Medicine and Industrial Hygiene, National Taiwan University, Taipei City 100, Taiwan; ${ }^{3}$ Department of Internal Medicine, Changhua Christian Hospital, Changhua 500, Taiwan; ${ }^{4}$ Department of Dentistry, Changhua Christian Hospital, Changhua 500, Taiwan; ${ }^{5}$ Graduate Institute of Statistics and Information Science, National Changhua University of Education, Changhua 500, Taiwan

\begin{abstract}
This study aimed to elucidate whether or not high-risk clusters of oral cancer $(\mathrm{OC})$ incidence spatially correlate with the prevalence rates of betel quid chewing (BQC) and cigarette smoking (CS) in Taiwan. The spatial autocorrelation and potential clusters of OC incidence among the 307 townships and heavy metal content of soil throughout Taiwan were identified using the Anselin's local Moran test. Additionally, the spatial correlations among the incidence of OC, the prevalence of BQC and CS and heavy metal content of soil were determined based on a comparison of spatial clusters. High-risk OC (Moran's $I=0.638, \mathrm{P}<0.001$ ) clusters were located in central and eastern Taiwan, while "hot spots" of BQC and CS prevalence were located mainly in eastern Taiwan. The distributions of BQC and CS lifestyle factors ( $\mathrm{P}<0.001)$ were spatially autocorrelated. The "hot spots" of OC largely coincided with the "hot spots" of BQC, except for the Changhua and Yunlin counties, which are located in central Taiwan. However, high soil contents of nickel and chromium $(\mathrm{P}<0.001)$ in central Taiwan also coincided with the high-risk areas of OC incidence. In particular, Changhua county has incurred several decades of serious heavy-metal pollution, with inhabitants living in polluted areas having high-risk exposure to these metals. Results of this study suggest that, in addition to BQC and CS, anthropogenic pollution may profoundly impact the complexity of OC aetiology in central Taiwan.
\end{abstract}

Keywords: spatial clustering analysis, geographical information system, oral cancer, aetiology, environmental pollution, Taiwan.

\section{Introduction}

Oral cancer (OC), characterised as tumor growth located in the mouth, is mainly of the squamous car-

Corresponding author:

Tsun-Kuo Chang

Department of Bioenvironmental Systems Engineering

National Taiwan University 1, Sec. 4, Roosevelt Road

Taipei City 106, Taiwan

Tel. +88623366 3466; Fax +886223631879

E-mail: tknchang@ntu.edu.tw cinoma type and is prevalent in South and Southeast Asia (Reichart and Way, 2006). Countries such as Bangladesh, India, Papua New Guinea and Taiwan have alarmingly high incidence rates of $\mathrm{OC}$, while the incidence and mortality rates of OC in Brazil and the United States of America have declined in recent decades (Boing et al., 2006; Kingsley et al., 2008). Conversely, in Taiwan, OC is among the fastest increasing malignancies with a 5.3-fold increase in the incidence of OC for males and a 2fold increase for females from 1982 to 2001 (Su et 
al., 2007). OC is more frequent in males and ranked as the fourth leading cause of cancer-related deaths in Taiwan since 2003 (DOH, 2003).

The International Agency for Research on Cancer (IARC), the World Health Organization's (WHO's) leading source for information about cancer (http://www.iarc.fr/) has identified intake of alcoholic beverages, betel quid chewing (BQC) with tobacco, and tobacco smoking as human carcinogens, with the target organs including the oral cavity, pharynx, larynx and esophagus (IARC, 1986, 1988). A statistically significant correlation between OC and BQC has been clearly demonstrated. As cigarette smoking (CS) and alcohol drinking play important roles in the aetiology of OC, these two activities may act synergistically (Blot et al., 1988; Merletti et al., 1989; Choi and Kahyo, 1991; Oreggia et al., 1991; Cancela et al., 2009). A casecontrol study in Taiwan revealed that the incidence of OC in patients who consumed tobacco, alcohol and betel quid was 123-fold higher than that of abstainers (Ko et al., 1995). However, as BQC and CS normally coexist in Taiwan, the effects of BQC on human health in Taiwan cannot be easily distin- guished from the integrated effects of both (Wen et al., 2005). Although the betel quid in Taiwan does not contain tobacco, the OC incidence in Taiwan is still higher than that in India, where most of the betel quid contains tobacco (Yang et al., 2005).

Advances in statistical methods involving disease mapping have created various research opportunities in fields such as environmental pollution and disease epidemiology. For example, it is now possible to easily present data cartographically as disease-specific maps, which facilitate the identification of possible factors related to the occurrence of disease as well as provide an additional perspective on clinical medicine, epidemiological studies and health improvement. A disease phenomenon clustered across space and time can be identified using cluster analysis approaches such as the Anselin's local Moran test (Rainey et al., 2006).

Consulting the Taiwan Cancer Database for the time period between 1995 and 2006, shows that the central part (Changhua and Yunlin counties) and eastern part (Taitung and Hualien counties) of Taiwan carry persistently high incidence rates of OC. However, as seen in Table 1, the male-to-female

Table 1. Annual age-standardised incidenze rate (ASIR) of male OC (female) and the average male-to-female ratios of OC incidence in central and eastern Taiwan and the rest of the counties 1995-2006.

\begin{tabular}{|c|c|c|c|c|c|c|c|c|c|c|}
\hline \multirow{3}{*}{$\begin{array}{l}\text { Year } \\
1995\end{array}$} & \multicolumn{4}{|c|}{ Central Taiwan } & \multicolumn{4}{|c|}{ Eastern Taiwan } & \multirow{2}{*}{\multicolumn{2}{|c|}{ Others $^{\mathrm{a}}$}} \\
\hline & \multicolumn{2}{|c|}{ Changhua } & \multicolumn{2}{|l|}{ Yunlin } & \multicolumn{2}{|c|}{ Taitung } & \multicolumn{2}{|c|}{ Hualien } & & \\
\hline & 28.36 & $(2.49)$ & 22.55 & $(1.70)$ & 25.58 & $(7.87)$ & 13.67 & $(4.34)$ & 13.34 & $(1.56)$ \\
\hline 1996 & 30.10 & $(2.50)$ & 25.64 & (1.13) & 30.57 & $(9.86)$ & 28.96 & $(4.23)$ & 14.26 & $(1.95)$ \\
\hline 1997 & 31.16 & $(1.96)$ & 36.34 & $(1.70)$ & 32.49 & (13.91) & 23.09 & $(7.11)$ & 16.70 & $(2.01)$ \\
\hline 1998 & 36.90 & $(1.75)$ & 29.87 & $(4.02)$ & 34.04 & $(4.64)$ & 28.41 & $(5.58)$ & 19.35 & $(2.09)$ \\
\hline 1999 & 40.66 & $(1.78)$ & 37.89 & (3.66) & 41.21 & (7.18) & 35.02 & $(5.66)$ & 20.71 & $(2.34)$ \\
\hline 2000 & 40.65 & $(2.49)$ & 37.01 & (3.18) & 39.53 & $(5.88)$ & 36.05 & $(5.10)$ & 22.87 & $(2.11)$ \\
\hline 2001 & 41.39 & $(2.15)$ & 38.42 & $(2.79)$ & 37.63 & $(14.46)$ & 24.63 & $(6.69)$ & 23.27 & $(2.55)$ \\
\hline 2002 & 40.65 & (3.59) & 36.69 & (1.87) & 42.81 & $(8.00)$ & 34.73 & $(3.55)$ & 23.65 & $(2.74)$ \\
\hline 2003 & 46.45 & $(3.32)$ & 55.81 & $(4.08)$ & 61.02 & (14.54) & 41.20 & $(5.99)$ & 27.25 & $(2.60)$ \\
\hline 2004 & 51.85 & $(3.12)$ & 54.01 & $(3.47)$ & 52.17 & (11.81) & 46.88 & $(6.39)$ & 28.85 & $(2.98)$ \\
\hline 2005 & 48.02 & $(2.86)$ & 51.43 & $(2.62)$ & 50.51 & (12.16) & 42.09 & $(7.50)$ & 29.18 & $(2.77)$ \\
\hline 2006 & 52.69 & $(3.58)$ & 60.07 & $(3.30)$ & 62.55 & $(12.92)$ & 51.81 & $(9.09)$ & 31.50 & $(2.89)$ \\
\hline Ratio $^{b}$ & 16.02 & & 15.60 & & 4.50 & & 5.88 & & 9.37 & \\
\hline
\end{tabular}

ASIR (per 100000 person-year); parentheses indicate age-standardised incidence rates of female OC.

${ }^{a}$ The average $\mathrm{OC}$ incidence rates for the rest of the 18 counties in Taiwan except for Changhua, Yunlin, Taitung and Hualien counties.

${ }^{b}$ The average male-to-female ratios of OC incidence for 12 years. The male-to-female ratios in other countries: Papua New Guinea (1.60), Sri Lanka (2.91), France (8.97), India (2.41), USA (2.14), Taiwan (9.47) and global average (2.55) in 2000 (Ferlay et al., 2001). 
ratio of OC incidence is around 15.81 in the central area (16.02 and 15.60 for Changhua and Yunlin counties, respectively) in contrast to that of 5.19 in the eastern area $(4.50$ and 5.88 for Taitung and Hualien counties, respectively) and the global average which is 2.55 (Ferlay et al., 2001). Thus, the cause of high OC incidence in these two areas may not be the same.

This study examines whether these previous findings, i.e. high OC incidence in central and eastern Taiwan, is statistically valid and whether other areas also have an elevated risk of OC. Additionally, geographical correlations of OC incidence with BQC and CS prevalence have been explored to evaluate the extent to which these two factors might explain the high incidence of OC reported as OC "hot spots".

\section{Materials and methods}

\section{Data on oral cancer patients}

Taiwan established its comprehensive cancer registry in 1979. It was followed by a compulsory health insurance system in 1995, which currently covers over $95 \%$ of its 23 million residents ( $\mathrm{Su}$ et al., 2007). The OC registration data from 1996 to 2002 were provided by Taiwan's Department of Health (DOH), which contains 19,535 male and 2,166 female patients diagnosed with malignant oral cavity cancer (ICD-O-FT T-140-141, 143-146, 148-149, see Appendix). The patients' homes were classified according to the residence code of each township. As Taiwan comprises 307 townships, each township was treated as a unit in the analysis in this study.

\section{Data on known risk habits of $\mathrm{OC}$}

The prevalence rates of BQC and CS were estimated from the National Health Interview Survey (NHIS) data in 2002 by Taiwan's DOH. The survey subjects included 1,086 males and 1,473 females. Similarly, data on the prevalence rates of BQC and CS were studied at the township level.

\section{Data on age-specific population in Taiwan}

The age-specific populations in each township from 1996 to 2002 were obtained from Taiwan's household registration system. The age-standardised incidence rate (ASIR) was based on the WHO world standard age-specific population for the year 2000 (Ahmad, 2000).

\section{Data on the content of heavy metals in soil}

Soil data were derived from a nationwide survey that determined the content in agricultural topsoil $(0-15 \mathrm{~cm})$ of arsenic (As), cadmium (Cd), chromium $(\mathrm{Cr})$, copper $(\mathrm{Cu})$, mercury $(\mathrm{Hg})$, nickel $(\mathrm{Ni})$, lead $(\mathrm{Pb})$ and zinc $(\mathrm{Zn})$, as obtained from the Environmental Protection Administration (EPA) in Taiwan from 1983 to 1986 (ROCEPA, 1989). The total concentration of extractable As and $\mathrm{Hg}$ in the soil was determined based on the aqua regia method, as well as the other six heavy metals by the $0.1 \mathrm{~N} \mathrm{HCl}$ extraction method. A grid cell size of 1,600 ha was used as a sampling unit and 936 soil samples were collected across Taiwan.

\section{Risk mapping and smoothing}

Excess-risk is the ratio of the observed OC incidence rate in each township to the average OC incidence rate of the entire study area. A value larger than one indicates that the township has a higher OC risk than the state average, while a value less than one signifies the opposite. Based on the excessrisk measure, a map can be constructed for evaluation of the risk of OC for each township. However, incidence rates of relatively rare diseases such as OC may be unstable. Therefore, an attempt was made to alleviate the variation of the OC incidence rate in administrative units with small populations by applying the spatial rate smoothing procedure. The procedure is based on the moving average or 'window' average of crude rates of each unit area, and a window is decided according to the k-nearest neighbour criterion (Fang, et al., 2006). In this study, 
$\mathrm{k}=6$ is used, i.e. the rates of exact 6 nearest neighbours of each unit (township) were combined to obtain the spatial-smoothed rate of that unit.

\section{Spatial statistical analyses}

A cluster-detection software programme, GeoDa0.9.5-I (Anselin, 2005) was used to test our hypothesis regarding spatial OC clusters in Taiwan. The null hypothesis for this study is that there is no spatial correlation in the OC incidence rates among neighbouring townships, while the alternative hypothesis is that such spatial correlation exists. The programme tested for spatial clustering was based on township area data, including OC case and at-risk population, as well as the location output, size and significance level of clusters.

The Moran Index (Moran's I) is a global spatial autocorrelation statistics method used to describe the overall spatial dependence of OC incidence over the study area. However, local spatial autocorrelation statistics (Moran's $I_{\mathrm{i}}$ ) could provide a determinant for each individual site, and it has also an attribute value correlated with the values in neighbouring sites. Specifically, the global Moran's I is a weighted average of local Moran's $I_{\mathrm{i}}$. Local autocorrelation analysis is performed based on the local indicator of spatial autocorrelation (LISA) statistics developed by Anselin (Anselin, 1995). The utility of this approach is that significant local homogeneous "hot spots", or heterogeneous "cold spots" areas, can be identified. The null hypothesis of the spatial autocorrelation test is that the $\mathrm{OC}$ incidence is not associated with neighbouring township levels, i.e. there is no spatial autocorrelation. An alternate hypothesis is that spatial clustering exists, i.e. neighbouring townships have a similar OC incidence. This study also defined a contiguity-based spatial weight, which was constructed for each township by queen contiguity relationships, which defines spatial neighbours as areas with a shared border and vertexes (Lai et al., 2009). For a statistical inference, 999 Monte Carlo permutations were performed with the significance level set as 0.001 .

\section{Results}

\section{Descriptive analysis}

From 1996 to 2002, 21,701 cases of OC were reported to the $\mathrm{DOH}$. The 7-year average annual raw incidence rates of OC in males and females per 100,000 person-years were 24.84 and 2.89 cases, respectively. After adjusting for age, the 7-year average annual ASIR were 25.74 male and 3.61 female cases per 100,000 person-years. The ASIR of male OC varied from 19.42 to 29.14 cases per 100,000 person-years, while the variation of female ASIR was between 2.85 and 4.45 cases per 100,000 personyears (Table 2). An increasing trend was observed in

Table 2. Annual and 7-year average annual incidence rates of OC in Taiwan 1996-2002.

\begin{tabular}{|c|c|c|c|c|c|c|c|c|}
\hline \multirow{2}{*}{ Year } & \multicolumn{4}{|c|}{ Male } & \multicolumn{4}{|c|}{ Female } \\
\hline & Case & At-risk population & Raw IR ${ }^{a}$ & $\mathrm{ASIR}^{\mathrm{b}}$ & Case & At-risk population & Raw IR $^{a}$ & ASIR $^{b}$ \\
\hline 1996 & 2,005 & $10,979,958$ & 18.26 & 19.42 & 261 & $10,383,277$ & 2.51 & 2.85 \\
\hline 1997 & 2,259 & $11,074,056$ & 20.40 & 21.55 & 271 & $10,498,429$ & 2.58 & 3.63 \\
\hline 1998 & 2,561 & $11,156,256$ & 22.96 & 26.06 & 278 & $10,606,759$ & 2.62 & 3.88 \\
\hline 1999 & 2,977 & $11,225,582$ & 26.52 & 28.15 & 323 & $10,701,168$ & 3.02 & 3.17 \\
\hline 2000 & 3,138 & $11,303,274$ & 27.76 & 27.03 & 314 & $10,804,881$ & 2.91 & 3.60 \\
\hline 2001 & 3,250 & $11,347,476$ & 28.64 & 28.80 & 369 & $10,879,049$ & 3.39 & 4.45 \\
\hline 2002 & 3,345 & $11,390,513$ & 29.37 & 29.14 & 350 & $10,950,071$ & 3.20 & 3.66 \\
\hline Average $^{c}$ & 2,791 & $11,211,016$ & 24.84 & 25.74 & 309 & $10,689,091$ & 2.89 & 3.61 \\
\hline
\end{tabular}

${ }^{\mathrm{a}} \mathrm{IR}=$ incidence rate (per 100,000 person-year); ${ }^{\mathrm{b}} \mathrm{ASIR}=$ age-standardised incidence rate (per 100,000 person-year); ${ }^{\mathrm{c}}$ Average $=$ the average values for 7 years, 1996-2002. 
the male OC incidence rate. It should be added that $90 \%$ of all OC cases in this study were males and only $10 \%$ females, which is a strong indicator of the predominance of OC among males in Taiwan.

\section{Spatial OC mapping in Taiwan}

Figure 1 displays a map depicting the distribution of the counties in Taiwan, while Figs. $2 a$ and $2 b$ illustrate the distribution of raw incidence and ASIR of male OC cases. An examination of Figs. $2 a$ and $2 \mathrm{~b}$ reveal aggregated areas representing a high OC incidence rate in Changhua, Yunlin, Taitung and Hualien counties, which are located in central and eastern Taiwan. According to the spatial smoothed map, central and eastern Taiwan apparently has a higher OC rate than other regions on the island (Fig. 2c). The distribution of excess-risk (Fig. 2d), indicates that central and eastern Taiwan are the main high-risk areas of male OC. Figure 3 displays the distribution of female $\mathrm{OC}$ incidence rate and the areas characterised by a high OC incidence rate in Hualien and Taitung counties, which are located in eastern Taiwan.

\section{Spatial clustering of OC incidence rate}

Spatial autocorrelation analyses for the 7-year average annual ASIR of OC in Taiwan from 1996 to 2002 revealed a significant global Moran's $I=0.638$ ( $\mathrm{P}<0.001)$, implying that the distribution of OC is spatially autocorrelated. Based on the global analy-

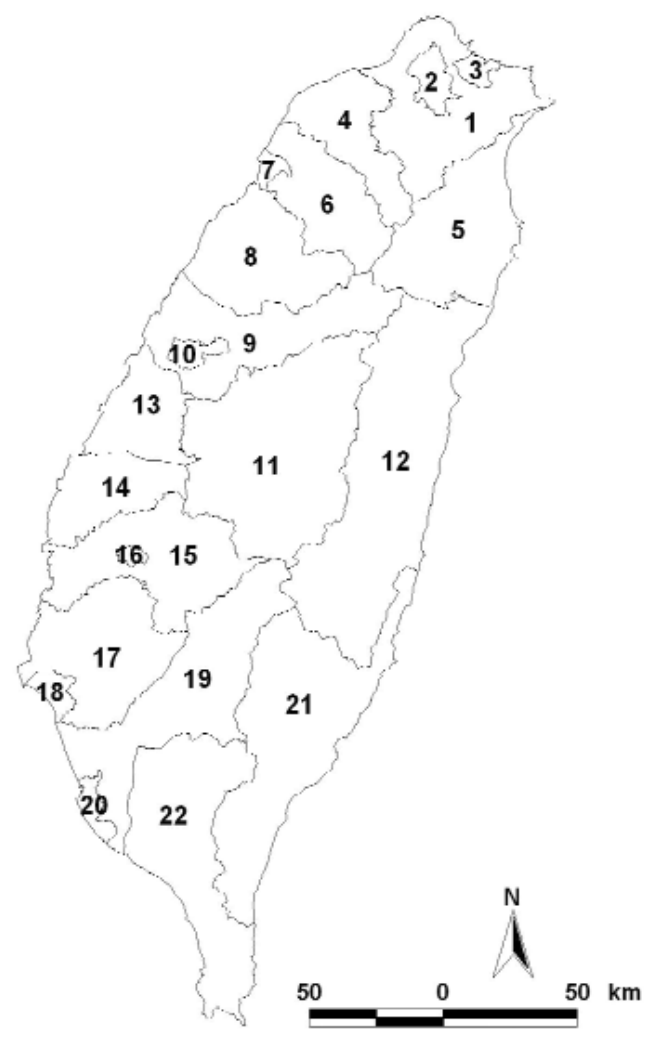

Fig. 1. Map of Taiwan at the county level. 1) Taipei county, 2) Taipei city, 3) Keelung county, 4) Taoyuan county, 5) Yilan county, 6) Hsinchu county, 7) Hsinchu city, 8) Miaoli county, 9) Taichung county, 10) Taichung city, 11, Nantou county, 12) Hualien county, 13) Changhua county, 14) Yunlin county, 15) Chiayi county, 16) Chiayi city, 17) Tainan county, 18) Tainan city, 19) Kaohsiung county, 20) Kaohsiung city, 21) Taitung county, 22) Pingtung county. 

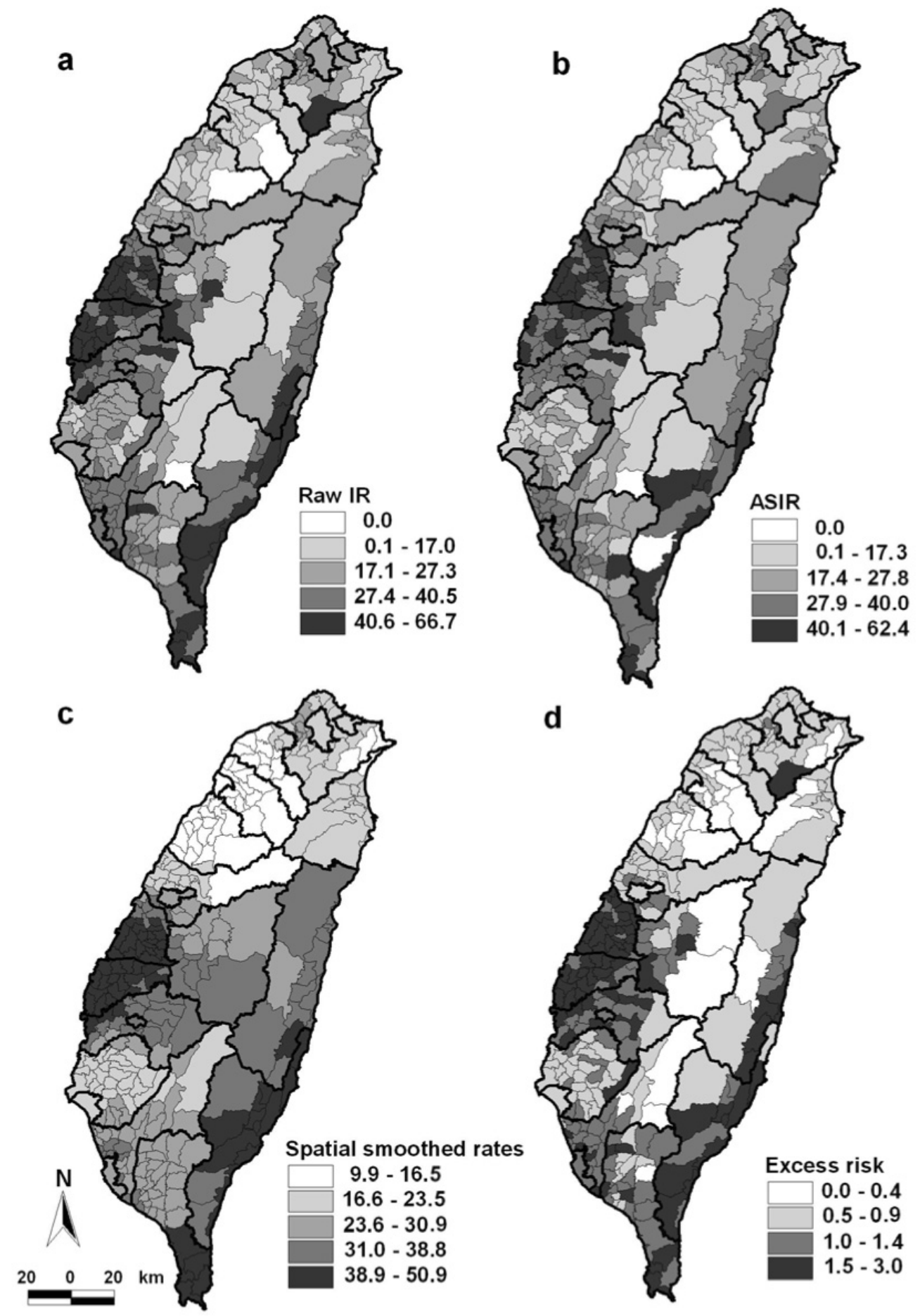

Fig. 2. Spatial distribution of incidence rate of male OC in Taiwan 1996-2002. a) Raw incidence rates (IR), b) age-standardised incidence rates (ASIR), c) spatial smoothed rates, and d) excess risk map. 

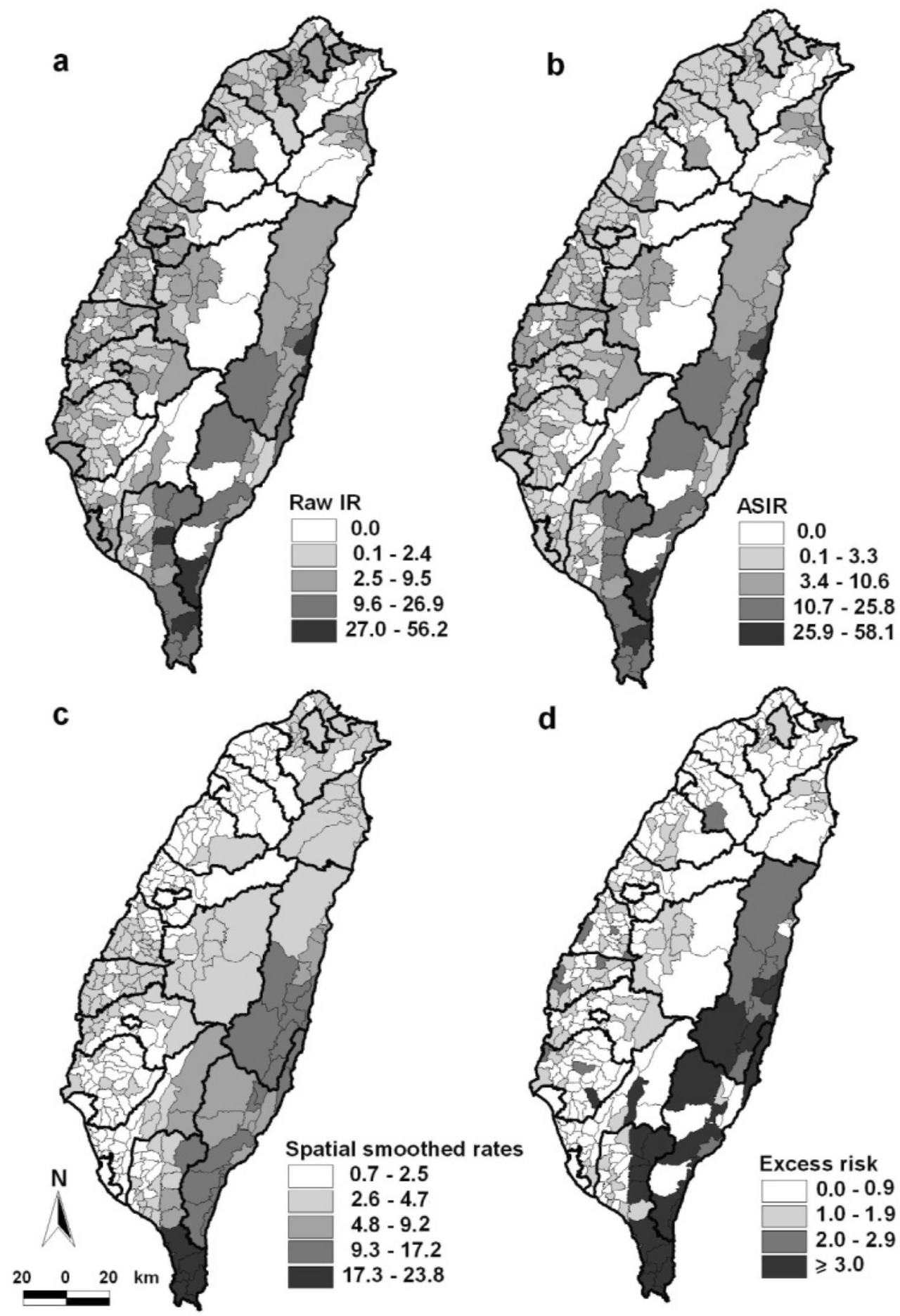

Fig. 3. Spatial distribution of incidence rate of female OC in Taiwan 1996-2002. a) Raw incidence rates (IR), b) age-standardised incidence rates (ASIR), c) spatial smoothed rates, and d) excess risk map. 
sis results, the "hot spots" were identified by performing the Local Moran test for spatial autocorrelation. The Local Moran test identified two major "hot spots" with a high ASIR similar to their neighbouring areas. The first high-risk OC cluster was located in central Taiwan on and around the Changhua and Yunlin counties. The second highrisk cluster was detected in Taitung county and Hualien county in eastern Taiwan (Fig. 4a).

\section{Spatial clustering of $B Q C$ and $C S$ prevalence rates}

As a determinant of global spatial autocorrelation, the global Moran's $I$ were both significant $(\mathrm{P}<0.001)$ for prevalence rates of BQC (Moran's $I=0.708$ ) and CS (Moran's $I=0.491$ ). Only one local homogenous "hot spot" with a high BQC prevalence rate was identified using LISA. The major "hot spot" of BQC prevalence rate was located in Taitung and Hualien counties in eastern Taiwan (Fig. 4b). As for the prevalence rate of CS, a single large area with a high CS prevalence rate was detected. Although the spatial cluster location of CS shifted upwards, as compared with the cluster location of BQC, it was still located mainly in eastern Taiwan (Fig. 4c). Unexpectedly, both identified potential clusters of high BQC and CS prevalence rates might not be exactly consistent in locations with the high-risk areas of OC identified by LISA.

\section{Spatial clustering of heavy metals in soil}

Figure 5 illustrates the spatial distribution of $\mathrm{Cr}$ and $\mathrm{Ni}$ content in soils throughout Taiwan from 1983 to 1986, as identified by the LISA test. Although the distributions of these eight heavy met-
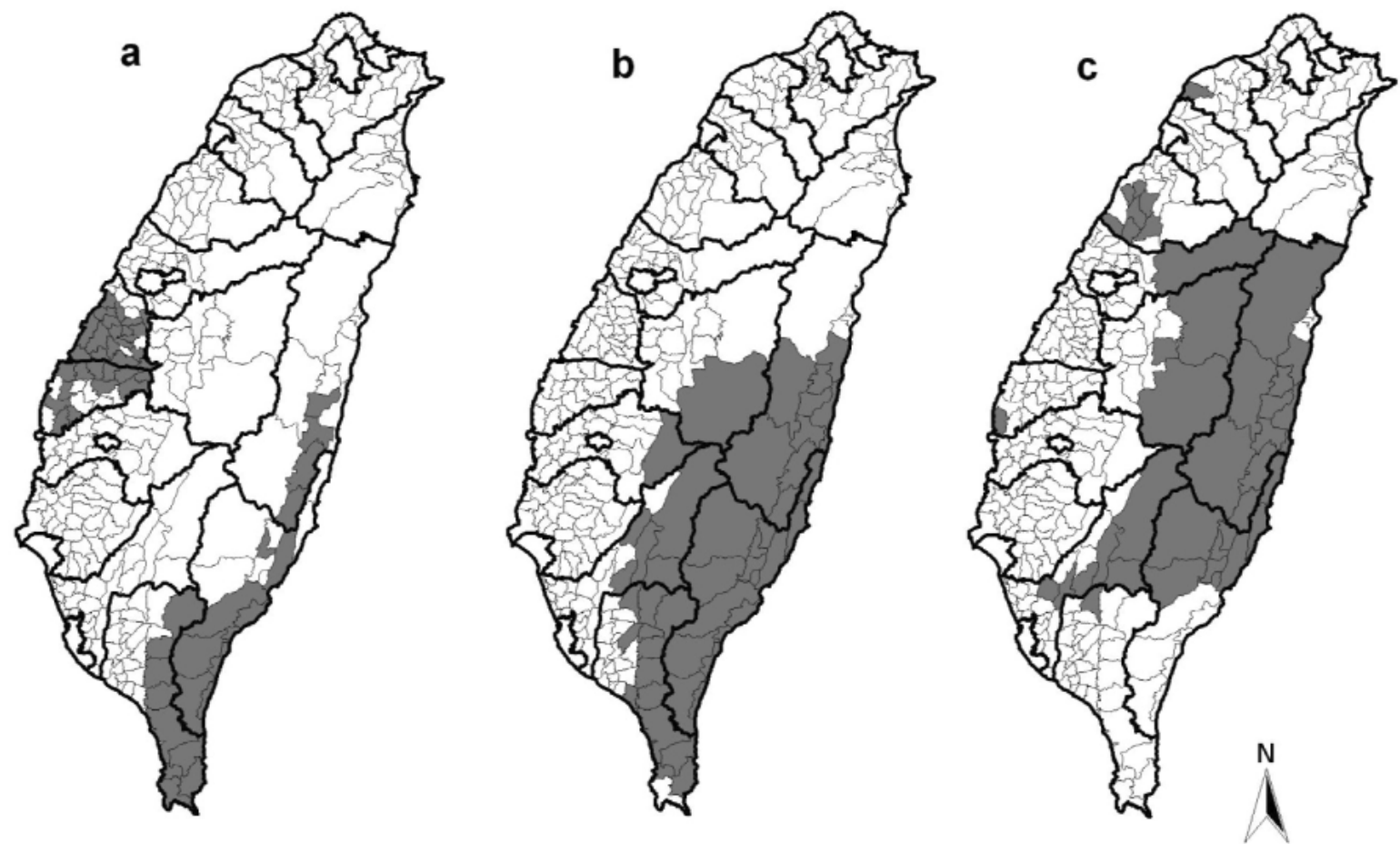

High-risk areas

$20 \quad 0 \quad 20 \mathrm{~km}$

Fig. 4. Statistically significant $(\mathrm{P}<0.01)$ high-risk clusters of $\mathrm{OC}$ incidence rate and the prevalence rates of $\mathrm{BQC}$ and $\mathrm{CS}$ in Taiwan identified by the Anselin's Local Moran test. a) OC incidence rate, b) BQC prevalence rate, c) CS prevalence rate. 
als (As, $\mathrm{Cd}, \mathrm{Cr}, \mathrm{Cu}, \mathrm{Hg}, \mathrm{Ni}, \mathrm{Pb}$ and $\mathrm{Zn}$ ) content were spatially autocorrelated $(\mathrm{P}<0.001)$ except for $\mathrm{Cd}$, the "hot spots" of high soil Cr (Moran's $I=$ 0.514 ) and $\mathrm{Ni}$ (Moran's $I=0.337$ ) content were clustered in central Taiwan.

\section{Discussion}

BQC and CS constitute established risk factors for OC in Taiwan. Many studies have demonstrated that $\mathrm{BQC}$ is the most important risk factor (Ko et al., 1995; Lu et al., 1996), but according to our results the aggregated areas of high BQC and CS prevalence rates were not the same as the geographic locations of high-risk OC. This finding does not necessarily contradict the results of other studies, which pointed to BQC and CS as the major culprits for OC. However, the observation implies that, although these two lifestyle factors might significantly impact the aetiology of OC, other aetiological or promoting factors, e.g. anthropogenic pollution, may also be pertinent for the onset of OC in Taiwan.

Studies in countries such as Australia, Taiwan, and the United States of America have demonstrated disparities in health between aboriginal and nonaboriginal individuals in a given population (Ko et al., 1994). BQC is a traditional aboriginal activity during festivals or ceremonial rituals in Taiwan. The whole population of 23 million includes various populations such as Hokkien (73\%), Hakka (12\%), Mainland Chinese $(13 \%)$, and aboriginal ethnic groups (2\%) (DOP, 1992). The latter reside mainly in the eastern valleys and central mountainous areas (Lin et al., 2008). In 2008, Hualien county was the home of $18.2 \%$ of the entire aboriginal population,
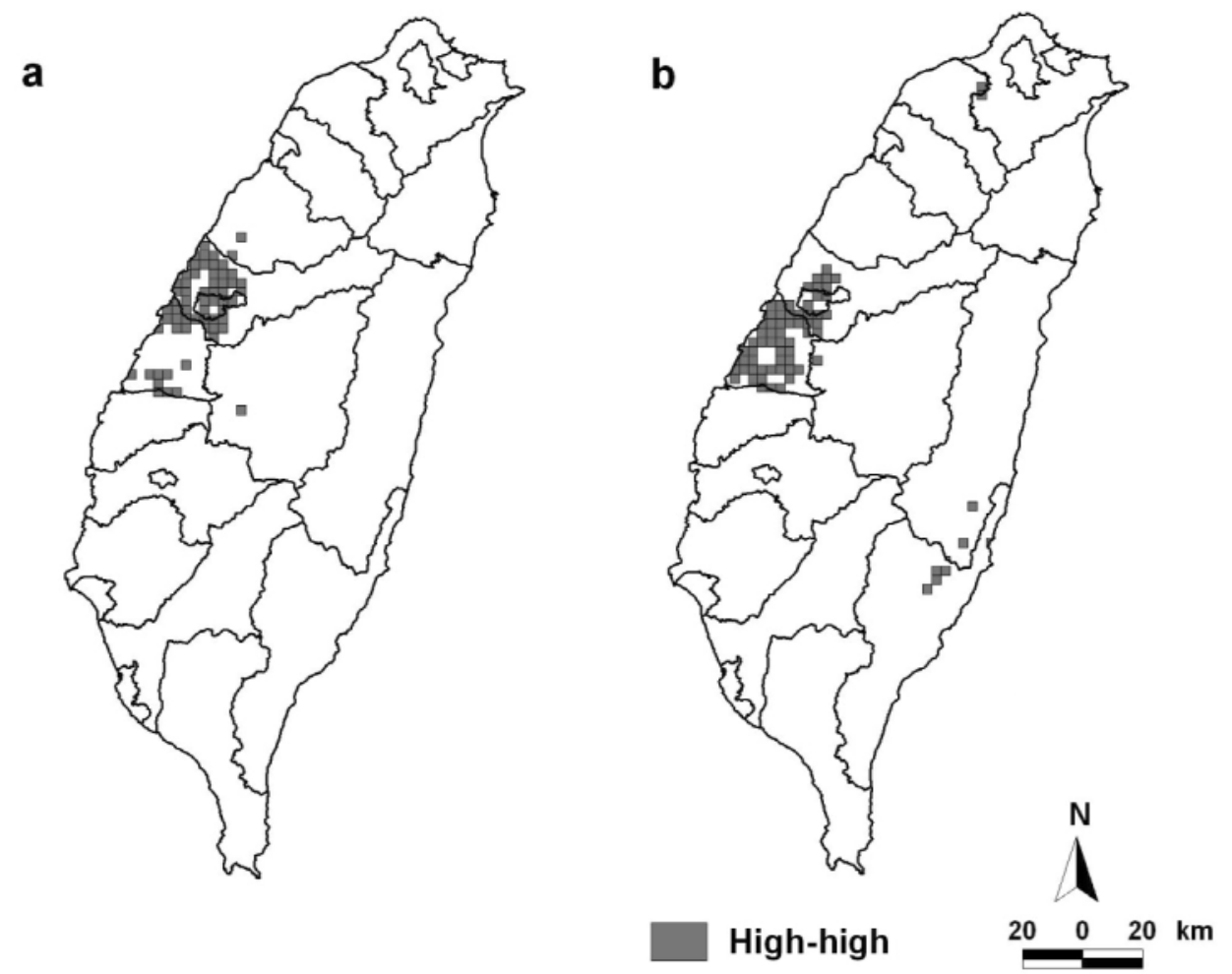

Fig. 5. Statistically significant $(\mathrm{P}<0.01)$ clusters of high chromium (a) and nickel (b) content. 
which accounts for the largest proportion of the aboriginal population on the island, followed by Taitung county with $16.0 \%$ (DHR, 2008). Taitung and Hualien counties, situated in the eastern part of Taiwan, have a high incidence of OC, and the BQC prevalence rates are high as well. Therefore, these two lifestyles largely account for the high-risk cluster of OC located in these two counties. In contrast, Changhua and Yunlin counties have less than $1 \%$ of the total aboriginal population. According to the results of the LISA cluster detection method, Changhua county, situated in central Taiwan, appears to belong to a high-risk OC area despite the fact that the prevalence rate of BQC in this county only ranked $11^{\text {th }}$ among 23 counties (Yang et al., 2002). However, most of the industrial plants for electroplating and other metal surface treatment are situated in this county. These types of factories are widely distributed throughout the area, possibly leading to serious heavy metal pollution of soil (Lin et al., 2002). A previous investigation of Changhua county indicates that the levels of chromium in whole blood (B-Cr) and urinary nickel (U-Ni) of local residents living in the factory-dense areas are higher than those in other areas (Chang et al., 2006a, b). Moreover, according to some studies, the blood nickel level of the non-occupationally exposed populations increased owing to environmental pollution (Linden et al., 1985; Demir et al., 2005). Thus, in addition to lifestyle factors such as BQC and CS, environmental factors might be linked to an increased risk of OC. Furthermore, the levels of female U-Ni were significantly higher than U-Ni level found in males (Chang et al., 2006b). Another study demonstrated that the mean concentrations of $\mathrm{Ni}, \mathrm{Cu}, \mathrm{Zn}$ and $\mathrm{Pb}$ in the sweat in healthy females were higher than those in males (Hohnadel et al., 1973). Females thus appear to have a better capability of excreting heavy metals from the body. If true, this may partially explain why the incidence of $\mathrm{OC}$ in females is substantially lower than in males in Changhua county, i.e. a male-to-female ratio where the highest values exceeded 16 .

Spatial analysis is obviously of value and it is believed that such methods will gradually become an integral component of epidemiological research and OC risk assessment. If a disease map were complemented with cluster detection, public health policymakers could better prioritize the specific areas where comprehensive investigations should be undertaken.

This study identified a high incidence of OC in central and eastern Taiwan, necessitating enhanced OC prevention and control measures, as well as efficient allocation of public health resources. Moreover, Changhua and Yunlin counties, i.e. highrisk areas for OC, could not be explained by BQC and CS only, or by ethnic differences. Therefore, further research is warranted with respect to the detected "hot spots" with the aim to identify the most important determinants for the incidence of OC in the high-risk areas in central Taiwan. Epidemiological evidence has been established in recent years on how exposure to heavy metals, e.g. $\mathrm{As}, \mathrm{Cr}$, and $\mathrm{Ni}$, and cancer are related (Hayes, 1997). In addition, experimental studies have clearly demonstrated that sodium dichromate dihydrate $(\mathrm{Cr}(\mathrm{VI})$ in drinking water causes oral cavity neoplasms in rats (Salnikow and Zhitkovich, 2008; Stout et al., 2009). Although our study found a higher incidence of OC risk in Changhua county than in any other area known to have a high OC incidence, it remains unclear whether or not environmental pollution increases the incidence of OC. However, according to our results, spatial distributions of heavy metals $\mathrm{Cr}$ and $\mathrm{Ni}$ in soils closely coincided with the spatial pattern of OC incidence. These clusters were similarly found in central Taiwan suggesting that a case-control study to compare contents of heavy metals in blood between OC and non-cancer patients is warranted as it would determine whether or not an increase in OC risk is related to specific heavy metals.

\section{Appendix}

ICD-O-FT (International Classification of Diseases for Oncology-Field Trial Edition) is used to code and classify anatomic site and histologic type 
of cancer cases. These codes denote: T-140, lip; T141, tongue; T-143, gum; T-144, floor of the mouth; T-145.2, T-145.3, T-145.5, palate; T-145.0, buccal mucosa; T-145.1, T-145.4, T-145.8, T-145.9, other and unspecified parts of mouth; T-146, oropharynx; T-148, hypopharynx; T-149, other and ill-defined sites in the lip, oral cavity and pharynx.

\section{Acknowledgements}

The authors would like to appreciate Taiwan's Department of Health for providing the cancer registry database. This research was financially supported by a grant from the National Science Council of the Republic of China, Taiwan (NSC 96-2314-B-018-001). Ted Knoy is appreciated for his editorial assistance.

\section{References}

Ahmad O, Boschi-Pinto C, Lopez AD, Murray CJL, Lozano R, Inoue M, 2000. Age standardization of rates: a new WHO standard (GPE Discussion Paper Series: No.31). World Health Organization, Geneva, Switzerland.

Anselin L, 1995. Local indicators of spatial association-LISA. Geogr Anal 27, 93-115.

Anselin L, 2005. GeoDaTM 0.9 User's Guide.

Blot WJ, McLaughlin JK, Winn DM, Austin DF, Greenberg RS, Preston-Martin S, Bernstein L, Schoenberg JB, Stemhagen A, Fraumeni JF, 1988. Smoking and drinking in relation to oral and pharyngeal cancer. Cancer Res 48, 3282-3287.

Boing AF, Peres MA, Antunes JL, 2006. Mortality from oral and pharyngeal cancer in Brazil: trends and regional patterns, 1979-2002. Rev Panam Salud Publica 20, 1-8.

Cancela MD, Ramadas K, Fayette JM, Thomas G, Muwonge R, Chapuis F, Thara S, Sankaranarayanan R, Sauvaget C, 2009. Alcohol intake and oral cavity cancer risk among men in a prospective study in Kerala, India. Community Dent Oral 37, 342-349.

Chang, FH, Wang SL, Huang YL, Tsai MH, Yu ST, Chang LW, 2006a. Biomonitoring of chromium for residents of areas with a high density of electroplating factories. J Expo Sci Environ Epidemiol 16, 138-146.

Chang FH, Wang HJ, Wang SL, Wang YC, Hsieh DP, Chang
LW, Ko YC, 2006b. Survey of urinary nickel in residents of areas with a high density of electroplating factories. Chemosphere 65, 1723-1730.

Choi SY, Kahyo H, 1991. Effect of cigarette smoking and alcohol consumption in the aetiology of cancer of the oral cavity, pharynx and larynx. Int J Epidemiol 20, 878-885.

DOH (Department of Health, Executive Yuan, R.O.C.), 2003. Cancer registry annual report of the Republic of China (Taiwan). Taipei, Taiwan.

DOP (Department of Population), 1992. Consensus 1990: Census report of the Republic of China (Taiwan). Taipei, Taiwan.

DHR (Department of Household Registration, Ministry of the Interior, R.O.C.), 2008. Demographics annual report of the Republic of China (Taiwan), Taipei, Taiwan.

Demir TA, Isikli B, Urer SM, Berber A, Akar T, Canbek M, Kalyoncu C, 2005. Nickel exposure and its effects. Biometals 18, 7-13.

Fang L, Yan L, Liang S, de Vlas SJ, Feng D, Han X, Zhao W, Xu B, Bian L, Yang H, Gong P, Richardus JH, Cao W, 2006. Spatial analysis of hemorrhagic fever with renal syndrome in China. BMC Infect Dis 6, 77.

Ferlay J, Bray F, Pisani P, Parkin DM, 2001. Globocan 2000: cancer incidence, mortality and prevalence worldwide, Lyon, France.

Hayes RB, 1997. The carcinogenicity of metals in humans. Cancer Cause Control 8, 371-385.

Hohnadel DC, Sunderma FW, Nechay MW, McNeely MD, 1973. Atomic absorption spectrometry of nickel, copper, zinc, and lead in sweat collected from healthy subjects during sauna bathing. Clin Chem 19, 1288-1292.

IARC, 1986. Betel-quid and areca-nut chewing, International Agency for Research on Cancer, Lyon, 141-291.

IARC, 1988. Alcohol drinking, International Agency for Research on Cancer, Lyon, 44 pp.

Kingsley K, O’Malley S, Ditmyer M, Chino M, 2008. Analysis of oral cancer epidemiology in the US reveals statespecific trends: implications for oral cancer prevention. BMC Public Health 8, 87.

Ko Y, Huang Y, Lee C, Chen M, Lin L, Tsai C, 1995. Betel quid chewing, cigarette-smoking and alcohol-consumption related to oral-cancer in Taiwan. J Oral Pathol Med 24, 450-453.

Ko YC, Liu BH, Hsieh SF, 1994. Issues on aboriginal health 
in Taiwan. Kaohsiung J Med Sci 10, 337-351.

Lai PC, So FM, Chan KQ, 2009. Areal methods of disease analysis. In: Spatial epidemiological approaches in disease mapping and analysis (Ed.). CRC press, Taylor \& Francis Group, 79 pp.

Lin CF, Shiau TJ, Ko YC, Chen PH, Wang JD, 2008. Prevalence and determinants of biochemical dysfunction of the liver in Atayal Aboriginal community of Taiwan: is betel nut chewing a risk factor? BMC Gastroenterol 8, 13.

Lin YP, Teng TP, Chang TK, 2002. Multivariate analysis of soil heavy metal pollution and landscape pattern in Changhua county in Taiwan. Landscape Urban Plan 62, 19-35.

Linden JV, Hopfer SM, Gossling HR, Sunderman FW, 1985. Blood nickel concentrations in patients with stainless-steel hip prostheses. Ann Clin Lab Sci 15, 459-464.

Lu CT, Yen YY, Ho CS, Ko YC, Tsai CC, Hsieh CC, Lan SJ, 1996. A case-control study of oral cancer in Changhua county, Taiwan. J Oral Pathol Med 25, 245-248.

Merletti F, Boffetta P, Ciccone G, Mashberg A, Terracini B, 1989. Role of tobacco and alcoholic beverages in the etiology of cancer of the oral cavity/oropharynx in Torino, Italy. Cancer Res 49, 4919-4924.

Oreggia F, De Stefani E, Correa P, Fierro L, 1991. Risk factors for cancer of the tongue in Uruguay. Cancer 67, 180183.

Rainey JJ, Omenah D, Sumba PO, Moormann AM, Rochford R, Wilson ML, 2006. Spatial clustering of endemic Burkitt's lymphoma in high-risk regions of Kenya. Int J Cancer 120, 121-127.

Reichart PA, Way TH, 2006. Oral cancer and pre-cancer in Myanmar: a short review. J Oral Pathol Med 35, 193-196.
ROCEPA (Environmental Protection Administration, Executive Yuan, R.O.C.), 1989. Survey of heavy metals in the soil samples. In: Statistic Office of Environmental Protection Administration (Ed.). Yearbook of environmental protection statistics Taiwan Area, the Republic of China, Environmental Protection Administration of the Republic of China, Taipei, Taiwan.

Salnikow K, Zhitkovich A, 2008. Genetic and epigenetic mechanisms in metal carcinogenesis and cocarcinogenesis: nickel, arsenic, and chromium. Chem Res Toxicol 21, 2844.

Stout MD, Herbert RA, Kissling GE, Collins BJ, Travlos GS, Witt KL, Melnick RL, Abdo KM, Malarkey DE, Hooth MJ, 2009. Hexavalent chromium is carcinogenic to $\mathrm{F} 344 / \mathrm{N}$ rats and B6C3F1 mice after chronic oral exposure. Environ Health Perspect 117, 716-722.

Su CC, Yang HF, Huang SJ, Lian IB, 2007. Distinctive features of oral cancer in Changhua county: high incidence, buccal mucosa preponderance, and a close relation to betel quid chewing habit. J Formos Med Assoc 106, 225-233.

Wen CP, Tsai SP, Cheng TY, Chen CJ, Levy DT, Yang HJ, Eriksen MP, 2005. Uncovering the relation between betel quid chewing and cigarette smoking in Taiwan. Tob Control 14, I16-I22.

Yang YH, Chen CH, Chang JS, Lin CC, Cheng TC, Shieh TY, 2005. Incidence rates of oral cancer and oral pre-cancerous lesions in a 6-year follow-up study of a Taiwanese aboriginal community. J Oral Pathol Med 34, 596-601.

Yang YH, Chen HR, Tseng YH, 2002. Prevalence rates of areca/betel quid chewing in counties of Taiwan. Taiwan J Oral Med Health Sci 18, 1-16. 